\title{
Aspectos cardiológicos relevantes en la infección COVID-19
}

\author{
David Martí Sánchez ${ }^{1, *}$, Concepción Fernández Pascual ${ }^{2}$, Alexander Felix Marschall ${ }^{3}$, Freddy \\ Andrés Delgado Calva ${ }^{4}$, Miriam Estébanez Muñoz ${ }^{5}$ y Salvador Álvarez Antón ${ }^{6}$ \\ 1 Servicio de Cardiología, Hospital Central de la Defensa Gómez Ulla, Universidad de Alcalá; \\ https://orcid.org/0000-0001-5036-4943 \\ 2 Servicio de Cardiología, Hospital Central de la Defensa Gómez Ulla, Universidad de Alcalá; \\ https://orcid.org/0000-0001-7424-1112 \\ 3 Servicio de Cardiología, Hospital Central de la Defensa Gómez Ulla, Universidad de Alcalá; \\ https://orcid.org/0000-0002-6024-0407 \\ 4 Servicio de Cardiología, Hospital Central de la Defensa Gómez Ulla, Universidad de Alcalá; \\ https://orcid.org/0000-0003-3084-7934 \\ 5 Servicio de Medicina Interna, Hospital Central de la Defensa Gómez Ulla, Universidad de Alcalá; \\ https://orcid.org/0000-0003-4509-9123 \\ 6 Servicio de Cardiología, Hospital Central de la Defensa Gómez Ulla, Universidad de Alcalá; \\ * Autor correspondencia: docalcala@hotmail.com
}

DOI: https://doi.org/10.37536/RIECS.2020.5.1.203

Recibido: 11/05/2020; Aceptado: 18/05/2020; Publicado: 30/05/2020

Resumen: Durante el mes de diciembre de 2019 se comunicaron en Wuhan, la capital de la provincia de Hubei, China, los primeros casos de neumonía asociada a la nueva variante de coronavirus 2 del síndrome respiratorio agudo grave (SARS-CoV-2). Durante el primer trimestre de 2020, la enfermedad por coronavirus de 2019 (COVID-19) experimentó una rápida expansión global, poniendo a prueba los sistemas sanitarios y financieros de las principales potencias del mundo. Paralelamente, el extraordinario esfuerzo en investigación ha permitido ampliar el conocimiento de la enfermedad, y en concreto de la afectación de otros órganos y sistemas más allá del aparato respiratorio. En la presente revisión se enumeran los aspectos clínicos más importantes en el manejo de los pacientes con COVID-19 desde el punto de vista cardiológico, desde la afectación miocárdica, al manejo de procesos cardiológicos específicos.

Palabras Clave: COVID-19, Troponina, Miocarditis, Arritmia.

\begin{abstract}
During December 2019, the first cases of pneumonia associated with the new variant of severe acute respiratory syndrome coronavirus 2 (SARS-CoV-2) were reported in Wuhan, the capital of Hubei province, China. During the first quarter of 2020, the 2019 coronavirus disease (COVID-19) underwent rapid global expansion, testing the health and financial systems of the world's leading powers. At the same time, the extraordinary research effort has allowed us to expand our knowledge of the disease, and specifically of the involvement of other organs and systems beyond the respiratory system. This review lists the most important clinical aspects in the management of patients with COVID-19 from the cardiological viewpoint, from myocardial injury to the management of specific cardiological procedures.
\end{abstract}

Key words: COVID-19, Troponin, Myocarditis, Arrhythmia. 
"No crecemos cuando las cosas son fáciles, crecemos cuando enfrentamos desafíos"

Nurma Clarkson

\section{Introducción}

La enfermedad por el nuevo coronavirus SARS-CoV-2 (COVID-19) ha supuesto, sin duda, el mayor desafío sanitario en varias décadas, reportándose casos en más de 200 países y territorios del mundo, y superándose ampliamente en los primeros meses de 2020 la cifra de 3 millones de infectados y 200.000 fallecidos [1].

El cuadro clínico característico consiste en una sintomatología viral de fiebre, tos y mialgias, acompañada o no de otras manifestaciones más infrecuentes y diversas, con evolución en algunos casos a un franco deterioro respiratorio típicamente después de la primera semana de síntomas, atribuido a la intensa respuesta inflamatoria del huésped [2,3].

El impacto tan grande sobre la salud pública ha venido acompañado de un gran esfuerzo en investigación clínica, que ha permitido reconocer la afectación de otros órganos y sistemas. En el presente artículo de revisión abordamos las distintas perspectivas de la relación COVID-19 y el sistema cardiovascular.

\section{Aspectos microbiológicos y clínicos generales de la infección COVID-19}

Los coronavirus son virus ARN monocatenarios positivos englobados dentro de la familia Coronaviridae. Estos virus son típicamente patógenos de animales vertebrados como aves y diferentes mamíferos, pero además tienen potencial de infección del ser humano [4].

Se han descrito siete coronavirus que infectan a los humanos, 4 de ellos son tipos comunes que dan lugar a cuadros leves (229E alfa coronavirus, NL63 alpha coronavirus, OC43 beta coronavirus y HKU1 beta coronavirus), y 3 son patógenos con capacidad de causar cuadros respiratorios graves (MERS-CoV, beta coronavirus del Síndrome Respiratorio del Medio Oriente o MERS; SARS-CoV, beta coronavirus del Síndrome Respiratorio Agudo Severo o SARS; y SARS-CoV-2, nuevo coronavirus que causa la enfermedad por coronavirus de 2019, o COVID-19).

Se ha postulado que el nuevo coronavirus es originario de murciélagos, adquiriendo la capacidad de infectar al hombre mediante mutaciones de ganancia de función dentro del dominio de unión al receptor [5]. Probablemente el aspecto más desafiante de la infección por SARS-CoV-2, además de su letalidad, es su tasa de reproducción básica más elevada que otros tipos de coronavirus o la gripe, en relación a la alta carga viral y lenta eliminación en personas infectadas, así como a la prolongada resistencia del virus en superficies contaminadas [6].

La unión del coronavirus al huésped se produce a través de las proteínas espiculadas (proteínas-S) que en su conjunto le dan la apariencia característica de "corona". Al igual que para SARS-CoV, el punto de unión es el receptor tipo 2 de la enzima convertidora de angiotensina 2 (ACE2). La ACE2 se expresa en los neumocitos tipo 2, que son el portal de entrada predominante. Adicionalmente, ACE2 se expresa en los cardiomiocitos, el epitelio intestinal, el endotelio vascular y los riñones, proporcionando un mecanismo para la disfunción multiorgánica que se puede ver con la infección por SARS-CoV [4,7].

La expresión de ACE2 se encuentra aumentada en procesos como la insuficiencia cardíaca, la hipertensión arterial o la aterosclerosis, a fin de contrarrestar la activación excesiva de angiotensina II, por lo que el virus podría ser más infectivo o letal en estos pacientes [8].

Por otra parte, comprender bien la interacción entre virus y huésped permite establecer dianas terapéuticas, y así actuando sobre la proteína $S$ o sobre la expresión de ACE2 podríamos eventualmente evitar la infección de la célula [4].

El periodo de incubación hasta la aparición de los primeros síntomas suele ser de 1 a 14 días (mediana, 6 días), y la sintomatología florida suele aparecer durante la $2^{2}$ semana desde el contacto (mediana de 11 días) [9]. La constelación de síntomas es amplia. La mayoría de pacientes presentan fiebre o tos seca, en ocasiones aparecen ageusia, anosmia o mialgias, y más raramente síntomas digestivos o molestias faríngeas. La disnea o fatiga severa aparecen a continuación de los síntomas iniciales, y se asocian con el desarrollo de neumonía. 
La radiografía simple de tórax es patológica en el 50-60\% de los pacientes y la tomografía computarizada en casi el $90 \%$ de los casos (figura 1). Los hallazgos más habituales son tenues infiltrados intersticiales, aumento difuso de densidad en vidrio deslustrado $u$ opacidades parcheadas, con frecuencia subpleurales, bilaterales o difusos, pero también focales. Al inicio no son típicos la franca consolidación con broncograma aéreo ni el derrame pleural. Los hallazgos analíticos característicos, que además han demostrado valor pronóstico, son la linfopenia absoluta, elevación de lactato deshidrogenasa, elevación de dímeros D, y elevación de ferritina $[2,3]$.
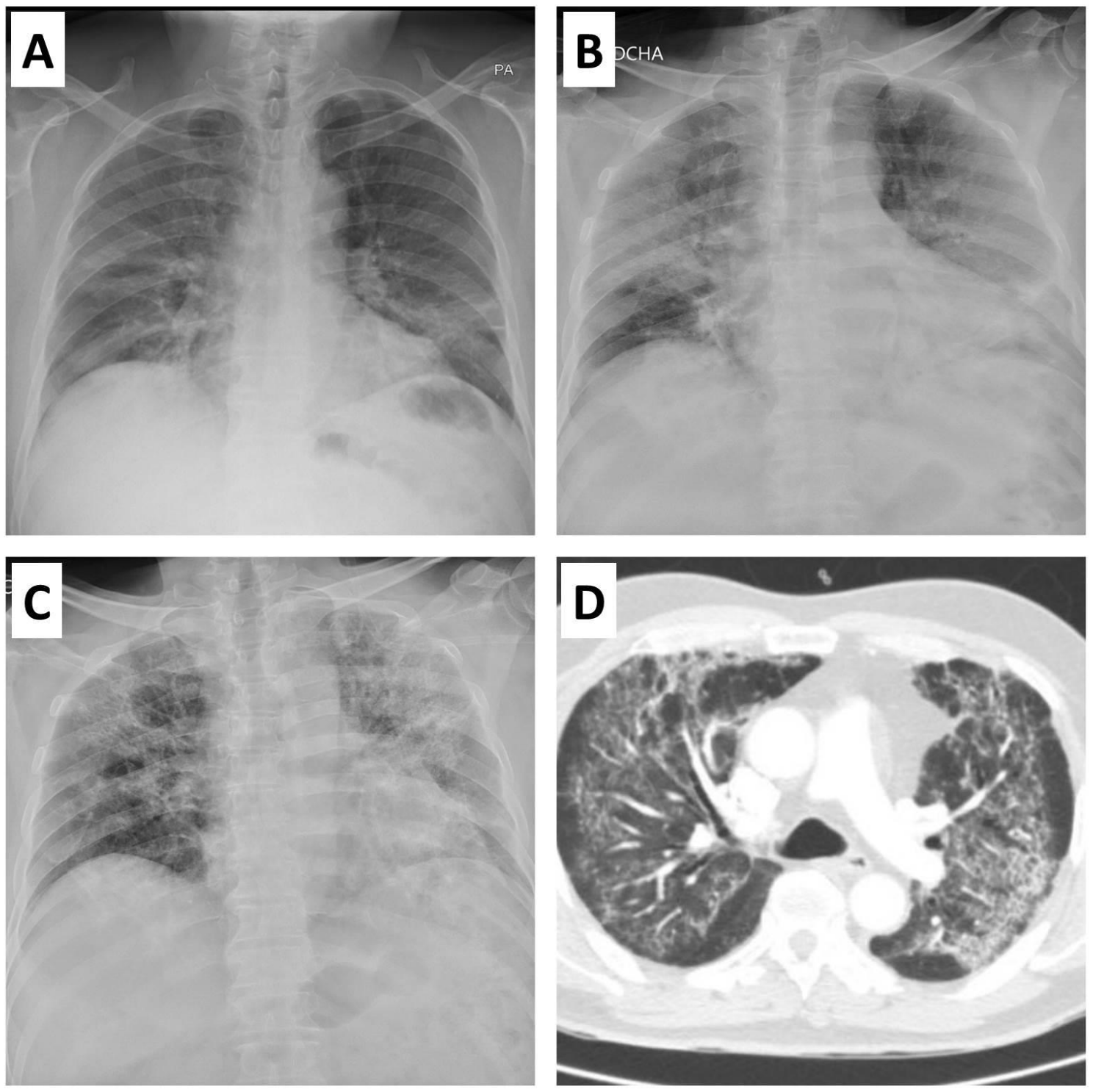

Figura 1 Evolución radiológica característica de varón de 65 años sin comorbilidades hospitalizado en nuestro centro por infección grave por SARS-CoV-2. A) Radiografía al ingreso (5o día de síntomas) que evidencia sutil patrón intersticial y zonas iniciales en vidrio deslustrado de distribución subpleural. B) Radiografía al $8^{\text {o }}$ día de síntomas, progresión de los cambios anteriores con zonas extensas de consolidación periférica. C) Radiografía al $33^{\circ}$ día de síntomas con persistencia de extenso patrón intesticial difuso con consolidaciones, áreas de fibrosis y bronquectasias. D) Tomografía computarizada torácica al 33ำ día de síntomas con engrosamiento de septos internos e intralobulillares asociado a extensas áreas en vidrio deslustrado de forma bilateral y difusa. Adicionalmente se aprecian pequeños defectos de repleción del contraste en arterías su segmentarías de ambos pulmones compatibles con trombosis en contexto de COVID-19. 
Si bien resulta difícil realizar estimaciones exactas, por diferencias en el estudio de los casos, en las características poblacionales y en los recursos sanitarios de las áreas afectadas, se estima que el $15-20 \%$ de las infecciones por SARS-CoV-2 dan lugar a cuadros clínicos graves, y según los informes de la Organización Mundial de la Salud la tasa de letalidad global se sitúa en torno al 7\% [1].

\section{Patología cardiovascular previa y COVID-19}

Las principales series publicadas se han centrado en pacientes hospitalizados con formas moderadas o graves de la enfermedad, y han evidenciado un cierto predominio masculino $(55-60 \%)$ y la afección predominante de pacientes en edad media de la vida (mediana de edad entre 47 y 56 años). En esta franja de edad, se ha reportado una prevalencia de comorbilidades de entre el 24$48 \%$, destacando por su frecuencia la hipertensión arterial (HTA, 15-30\%), la diabetes mellitus (7$19 \%$ ) y las enfermedades cardiovasculares (ECV, 3-15\%) [2,3,11-13].

Es difícil establecer el efecto exacto de la ECV sobre la mortalidad por COVID-19, dada su estrecha relación con la edad avanzada, que es probablemente el aspecto que más influencia tiene en el desenlace clínico. Sin embargo, algunos estudios han sugerido que las condiciones cardiovasculares preexistentes son predictores importantes de la gravedad de COVID-19.

En un análisis de los 72.314 pacientes con infección confirmada o probable reportados en China hasta febrero de 2020, la tasa de mortalidad total fue del 2,3\%, cifrándose en el 0,9\% en pacientes sin comorbilidades, en torno al 6-7\% en pacientes con hipertensión, diabetes, enfermedad respiratoria o cáncer, y en el 10,5\% en aquellos con ECV. La aparición de ECV afectaría la tasa de mortalidad en mayor medida que la enfermedad pulmonar obstructiva crónica, y la mayor edad poblacional y prevalencia de ECV podrían en parte justificar la mayor letalidad del virus en determinadas regiones geográficas $[12,14]$.

Llamativamente, en un estudio del Séptimo Hospital de Wuhan, la mayor mortalidad se concentró en los pacientes que además de ECV presentaron elevación de troponina sugestiva de daño miocárdico agudo, mientras que aquellos con ECV pero troponina normal presentaron un pronóstico más favorable (mortalidad del $69 \%$ frente al 13\% respectivamente) [13].

Una mención especial merece la relación entre la HTA y la infección por SARS-CoV-2. Los datos disponibles muestran tasas de prevalencia concordantes con las de la población general $(\sim 30 \%)$, por lo que no parece que los pacientes hipertensos tengan mayor susceptibilidad a la infección [15]. Más probable es la relación entre HTA y severidad de la enfermedad, y la mayoría de los estudios han mostrado un incremento del riesgo de deterioro clínico o de mortalidad en los sujetos hipertensos [2,14]. Sin embargo, una vez más esta asociación puede estar influenciada por la elevada prevalencia de hipertensión en las personas mayores, quienes presentan una evolución significativamente menos favorable [12]. En general, es esencial optimizar el control de la presión arterial en pacientes hipertensos durante el COVID-19, se desaconsejan los cambios innecesarios y controvertidos en las terapias, y los pacientes hipertensos deben ser vigilados cuidadosamente para detectar complicaciones durante la infección por COVID-19. 


\section{Manifestaciones cardiovasculares de COVID-19}

El COVID-19 se asocia característicamente a una "tormenta de citoquinas" que desemboca en la inflamación sistémica progresiva, el fallo multiorgánico y la muerte. En este contexto, varios factores se han asociado a la afectación cardiaca, además de la inflamación, fenómenos de trombosis e hipercoagulabilidad, incremento del tono adrenérgico, regulación negativa de los receptores ACE2, o efectos de la medicación [16].

Entre las manifestaciones cardiovasculares de la infección hay que destacar el daño miocárdico agudo y la miocarditis, las arritmias, y fenómenos trombóticos como los síndromes coronarios agudos o la tromboembolia de pulmón (figura 2).

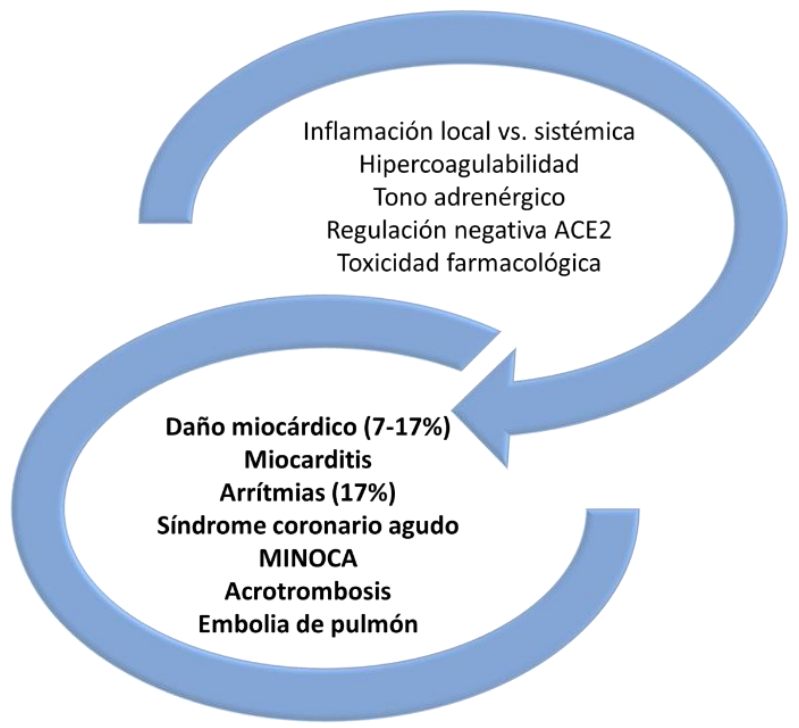

Figura 2 Esquema de mecanismos posibles y manifestaciones cardiovasculares de la infección por SARS-CoV-2. MINOCA: infarto de miocardio sin lesiones coronarias obstructivas.

\subsection{COVID-19, lesión miocárdica y miocarditis}

Casi todos los pacientes hospitalizados muestran niveles elevados de creatina quinasa (CK) y lactato deshidrogenasa (LDH) que sugieren un componente de daño muscular. Adicionalmente, los principales estudios publicados han puesto de manifiesto una elevación significativa de troponina cardiaca en el 7-17\% de los pacientes [4]. Zhou y colaboradores reportaron elevación de troponina I de alta sensibilidad (hs-cTnI $>28 \mathrm{pg} / \mathrm{mL}$ ) en el $46 \%$ de pacientes que fallecieron, frente a únicamente el $1 \%$ de los pacientes que sobrevivieron al ingreso hospitalario [3]. En el estudio de Guo y cols., la mortalidad de los pacientes con elevación de Troponina T $(>99 \%$ del límite superior de la normalidad) osciló entre el $37 \%$ en pacientes sin ECV previa, y el $69 \%$ en pacientes con ECV previa [13].

Se han descrito dos patrones característicos de lesión miocárdica en el contexto de COVID-19 [7]. La mayoría de los pacientes experimentan un daño progresivo en el seno de la tormenta citoquímica tal y como se observó en el estudio de Zhou, en el que la mediana de hs-cTnI se mantuvo prácticamente normal e inalterada en supervivientes, mientras que aumentó progresivamente desde el día 7 en no supervivientes. En estos, la mediana de hs-cTnI fue 8,8 pg/mL al ingreso, 24,7 pg / $\mathrm{mL}$ al $7^{0}$ día, a 290,6 pg / $\mathrm{mL}$ al 22ํㅜ día, encontrándose esta evolución relacionada con la de los mediadores inflamatorios (dímero D, ferritina, interleucina-6, lactato deshidrogenasa) [3].

Por el contrario, algunos pacientes desarrollarían un cuadro de miocarditis con elevación de la troponina desde los primeros días de ingreso [17]. Aunque aún no se ha realizado de forma sistemática biopsia endomiocárdica para la identificación de partículas virales, una explicación factible sería la infección directa del virus seguida de la respuesta inflamatoria del huésped. Se han 
comunicado casos de miocarditis fulminante con sintomatología predominantemente cardiaca (shock cardiogénico, dolor torácico sin obstrucción coronaria) y severa afectación de la función ventricular, así como recuperación con inmunoglobulina intravenosa y esteroides a dosis altas [18].

En síntesis, los mecanismos subyacentes a la lesión miocárdica parecen diversos, y responderían a inflamación sistémica, infección directa del cardiomiocito mediada por ACE2, trombosis o disbalance entre aporte y demanda, e incluso efecto de los tratamientos. Se puede interpretar la lesión miocárdica como marcador o factor de riesgo de mortalidad por COVID-19, por lo es importante monitorizar los niveles de troponina en las formas graves de la enfermedad, observándose en la mayoría de los casos la elevación a partir de los días 7-10 de ingreso [4,13].

\subsection{COVID-19 y arritmia cardiaca}

Como se ha observado en otras infecciones virales, tanto la afectación de la función cardiaca como las alteraciones metabólicas y autonómicas pueden favorecer la aparición de arritmias [16]. En el estudio de Wang y cols. se observó alguna arritmia en 1 de cada 6 pacientes ingresados por COVID-19, en concreto en el $44 \%$ de los que requirieron tratamiento en UCI y el $7 \%$ de los que permanecieron en planta de hospitalización [11]. Guo y cols. registraron una incidencia de arritmias ventriculares malignas (taquicardia ventricular o fibrilación ventricular) del 6\%, particularmente en pacientes con elevación de troponina cardiaca [13]. Por último, es importante enfatizar el potencial proarrítmico de la medicación específica, que se abordará en detalle más adelante.

\subsection{Sindromes coronarios agudos}

Si bien la enfermedad viral y la inflamación pueden desestabilizar las placas ateroscleróticas, los informes iniciales indican que la mayoría de los casos corresponden a infarto de miocardio sin lesiones coronarias obstructivas (MINOCA del acrónimo en inglés) [4]. En una comunicación personal de 34 pacientes tratados en la región de Lombardía, en el 62\% no se identificó una lesión trombótica culpable. El mecanismo en estos casos podría ser un infarto "tipo 2" (disbalance entre demanda y aporte) o la infección directa de los pericitos con inflamación y trombosis microvascular. Debido a lo anterior, la decisión para el manejo invasivo versus no invasivo de los síndromes coronarios agudos debe considerarse cuidadosamente.

\subsection{Estado protrombótico durante la infección por COVID-19}

La infección por SARS-CoV-2 se asocia a niveles elevados de dímero D y productos de degradación de la fibrina, y la experiencia de China indica que un aumento del dímero $\mathrm{D}$ es altamente predictivo de resultados adversos. El riesgo de embolia de pulmón, trombosis microvascular y coagulación intravascular diseminada se encuentran elevados en estos pacientes, pero aún es controvertido el incremento del riesgo en comparación al de otras enfermedades críticas, así como sus potenciales implicaciones terapéuticas [4,19]. La mayoría de los protocolos hospitalarios contemplan la administración de dosis profilácticas de heparina de bajo peso molecular durante la hospitalización y durante las semanas de duración del periodo de aislamiento tras el alta hospitalaria.

\subsection{Efectos crónicos sobre el sistema cardiovascular}

El seguimiento a 12 años de los pacientes recuperados de la infección por SARS-CoV reveló anomalías del sistema cardiovascular en el $44 \%$, y alteraciones lipídicas o glucémicas en el $68 \%$ y $60 \%$ respectivamente [20]. Parece que la infección viral conduce a trastornos del metabolismo de los lípidos y la glucosa por mecanismos aún inciertos. Dado que el SARS-CoV-2 tiene una estructura similar al SARS-CoV, este nuevo virus también podría causar daño crónico al sistema cardiovascular, por lo que se debe enfatizar la prevención del riesgo vascular a largo plazo en estos pacientes. 


\section{Efectos de la medicación frente a COVID-19 en el sistema cardiovascular}

El tratamiento contra la infección por COVID-19 ha supuesto uno de los principales retos de esta pandemia, no solo por el intento apresurado de frenar el avance de la enfermedad, sino por la posible aparición de efectos secundarios.

Uno de los riesgos más preocupantes es el potencial de arritmias, secundario al efecto cardiotóxico de algunos de estos fármacos, añadido a la propia fisiopatología de la infección por COVID-19 y a la interacción con los fármacos empleados para el tratamiento de otras patologías $[21,22]$.

\subsection{Efectos crónicos sobre el sistema de conducción}

Tanto el lopinavir-ritonavir como la hidroxicloroquina pueden prolongar el QT y el intervalo $\mathrm{PR}$, especialmente en pacientes que basalmente presentan dicha alteración o en aquellos sujetos que toman fármacos prolongadores del QT (figura 3). Las consecuencias de ello serían el desarrollo de taquicardia ventricular polimorfa en "torsión de puntas" o bloqueo auriculoventricular respectivamente.

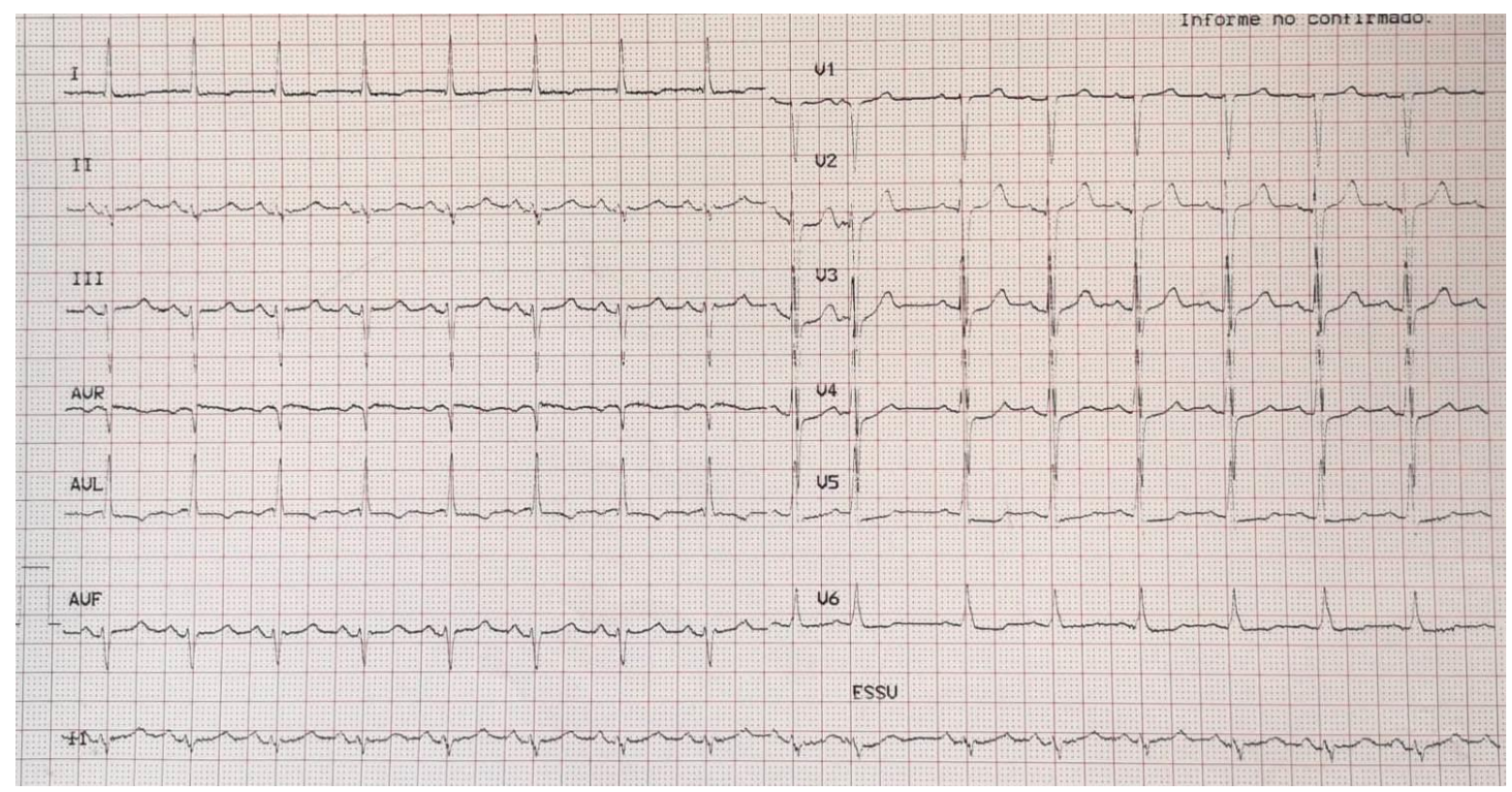

Figura 3 Prolongación del intervalo QT en paciente con tratamiento combinado con hidroxicloroquina y azitromicina. El eje izquierdo sugiere cardiopatía predisponente como factor de riesgo de QT largo. El intervalo debe medirse en las derivaciones en las que esté más prolongado, habitualmente DII o V5, en este caso el QT corregido es $490 \mathrm{mseg}$ (normal $<460 \mathrm{mseg}$ ).

De igual modo, la azitromicina también se encuentra entre los fármacos que pueden prolongan en QT, con el subsecuente riesgo de arritmias ventriculares.

Puesto que los tres fármacos implicados fundamente en el tratamiento para el COVID-19 tienen un potencial efecto arritmogénico, se han desarrollado múltiples protocolos (figura 4), que de forma genérica podrían resumirse de la siguiente manera:

- Inicialmente se debe realizar una valoración del paciente identificando la presencia de factores no modificables (edad >65años, antecedentes de cardiopatía estructural, síndrome de QT largo congénito, enfermedad renal o hepática), así como los tratamientos basales que puedan prolongar el intervalo QT (disponible en: http://www.qtdrugs.org). Del mismo modo hay que realizar un ECG basal y una analítica para confirmar que iones como el potasio, magnesio y calcio están en rango.

- Una escala frecuentemente utilizada para pacientes hospitalizados es la propuesta por Tisdale y cols., que permite clasificar como alto o bajo el riesgo de presentar prolongación del QT, y en 
función de lo cual monitorizar el ECG una vez iniciado el tratamiento. A falta de un documento oficial de consenso, parece razonable partir de un valor de referencia de QT corregido (QTc) de $460 \mathrm{mseg}$, y realizar una seriación a las 24 horas de inicio del tratamiento, en caso de que el QTc permanezca inferior a esa cifra, podrá hacerse una vigilancia más laxa con ECG y analítica cada 72 horas. Cuando a las 24 horas del inicio del tratamiento el QTc se encuentre entre 460 y $500 \mathrm{mseg}$, la monitorización deberá hacerse cada 48 horas. Para aquellos casos en los que la prolongación sea igual o mayor a $500 \mathrm{mseg}$, o se haya incrementado 60 mseg o más respecto al basal deberá hacerse una determinación inmediata de iones sanguíneos (asegurando un potasio sérico $>4 \mathrm{mEq} / \mathrm{l}$ y un magnesio $>2 \mathrm{mEq} / \mathrm{l}$ ) con una monitorización cada 24 horas de ECG y analítica, así como una valoración conjunta con cardiología del riesgobeneficio de mantener el tratamiento.

- Es importante prestar especial atención a la presencia de extrasístoles ventriculares o al desarrollo de síncope, como datos de alarma a tener en cuenta para la retirada de estos fármacos.

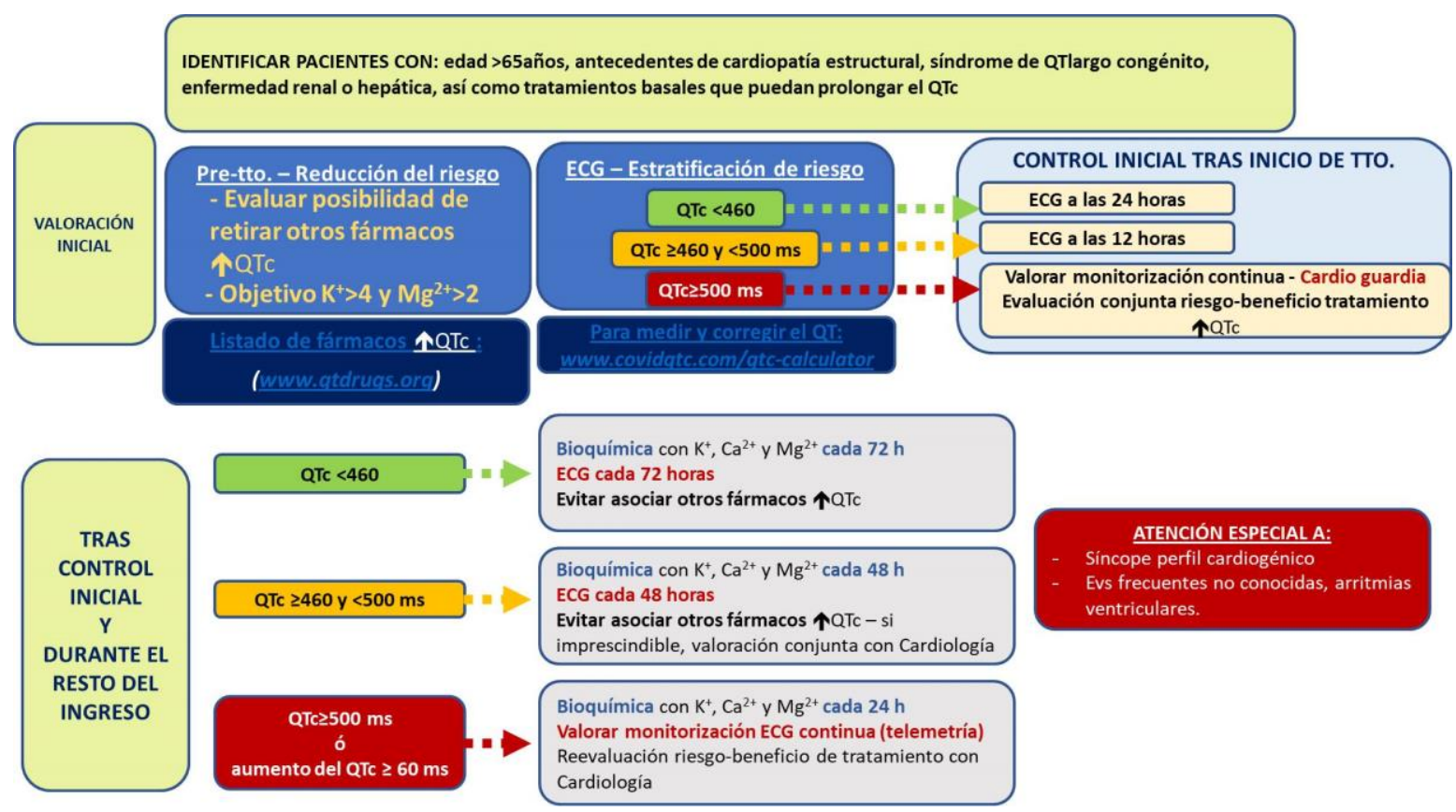

Figura 4 Protocolo de monitorización de QT del Hospital Central de la Defensa, Madrid. Para explicación, remitirse al texto.

\subsection{Toxicidad sobre el miocardio}

La hidroxicloroquina puede producir miocardiopatía dilatada o restrictiva, así como exacerbar una miocardiopatía ya existente (si bien esto es reproducible sobretodo en tratamientos de larga duración).

\subsection{Hipertensión arterial}

Se ha descrito esta posible complicación en pacientes bajo tratamiento con tocilizumab. Así mismo, los corticoides pueden alterar la mecánica hidroelectrolítica favoreciendo a un aumento en la retención de fluidos, y por tanto incrementar de la tensión arterial. 


\section{Consideraciones respecto a las terapias cardiológicas en el paciente con infección COVID-19}

\subsection{Interacciones con fármacos de uso frecuente en cardiología}

Existen un amplio número de interacciones farmacológicas que han sido recogidas por la Universidad de Liverpool en un documento ampliamente difundido (disponible en: https://www.covid19-druginteractions.org/). De forma resumida y orientada a grupos farmacológicos, destacamos las más importantes.

\subsubsection{Lopinavir-Ritonavir:}

- Fármacos anticoagulantes orales: debido a la interacción con el CYP3A4 y glicoproteína-P, se aconseja discontinuar o reducir dosis de los fármacos antiXa (apixaban, rivaroxaban y edoxaban). Deberán usarse con precaución dabigatrán y antagonistas de la vitamina $\mathrm{K}$ (acenocumarol/warfarina), estos últimos con controles frecuentes de INR.

- Fármacos antiagregantes: La inhibición del CYP3A4 hace disminuir el metabolito activo de clopidogrel y prasugrel, y aumenta las concentraciones plasmáticas de ticagrelor. En general y pese a la escasa evidencia disponible, se recomienda prasugrel, y en caso de contraindicación al mismo, valorar terapia individualizada mediante tests de antiagregación plaquetaria [21].

- Estatinas: La administración conjunta produce un aumento de la concentración del hipolipemiante, con mayor riesgo de mialgias o rabdomiolisis. Se recomienda la reducción de dosis de rosuvastatina y atorvastatina a un máximo de 10 y $20 \mathrm{mg}$ al día respectivamente.

- Antiarrítmicos: Conforme a lo expuesto en el apartado anterior, hay que tener especial atención a la administración de fármacos que prolonguen el QT (antiarrítmicos de clases Ia y III). Existe también un mayor riesgo de intoxicación digitálica por lo que se aconseja vigilar los niveles de digoxina en sangre.

- Hidroxicloroquina: Además del efecto proarrítmico, debido a la inhibición de CYP2D6 pueden verse incrementados los niveles de algunos betabloqueantes (metoprolol, carvedilol, propanolol o labetalol) [21].

- Corticoides: Se ha descrito interacción con warfarina/acenocumarol, por lo que hay que tener especial cuidado en la administración conjunta.

\subsection{Recomendaciones actuales de los fármacos bloqueadores del sistema renina-angiotensina-aldosterona en pacientes con COVID-19}

Es importante dedicar un apartado específico a este grupo farmacológico por las múltiples referencias al mismo durante los primeros meses de pandemia.

La sospecha no demostrada de que una mayor expresión de ECA2 en las células pulmonares de pacientes bajo tratamiento con IECA/ARA II, generó una hipótesis inicial sobre su posible susceptibilidad a padecer infección grave por COVID-19. Frente a ello, diversas publicaciones posteriores no han encontrado asociación entre la severidad de la infección viral con la toma de estos $\mathrm{u}$ otros antihipertensivos, e incluso algunos estudios han postulado un posible efecto protector $[12,24,25]$. Si bien es cierto que un porcentaje de los pacientes con COVID-19 se encuentran bajo tratamiento con IECA/ARA II, ello es debido a la prevalencia de ECV en estos pacientes. Por todo ello, las distintas sociedades científicas, incluida la Sociedad Europea de Cardiología, han sido unánimes en no recomendar la retirada de estos fármacos, hecho que lejos de ser beneficioso podría ser perjudicial para los pacientes con hipertensión arterial o insuficiencia cardiaca. 


\subsection{Anticoagulación en paciente con COVID-19}

En este aspecto se plantean dos escenarios concretos. Por un lado, observamos pacientes con indicación de anticoagulación previa y que ingresan por COVID-19. En estos casos debemos tener en cuenta las interacciones descritas con los anticoagulantes orales, procediendo a los pertinentes ajustes de dosis, o sustituyéndolos temporalmente por heparina de bajo peso molecular (HBPM) dada su ausencia de interacciones relevantes durante el periodo de tratamiento antiviral activo.

El segundo escenario corresponde a los pacientes en los que iniciamos tratamiento anticoagulante durante la pandemia por COVID-19. En estos casos hay que tener en consideración la necesidad de aislamiento o confinamiento domiciliario, con eventual dificultad para control de INR en el periodo inicial de mayor riesgo de ictus. Por ello, distintas comunidades autónomas han facilitado el acceso a los anticoagulantes orales de acción directa (ACOD). En pacientes que tengan contraindicación para ACOD habrá que optar de forma individualizada entre mantener con HBPM a dosis anticoagulantes o el inicio de antagonistas de la vitamina $\mathrm{K}$, con las peculiaridades que ello implica en cada uno de los casos [26].

\section{Gestión de la patología cardiovascular en el contexto de la pandemia}

Sin duda, uno de los efectos de la presente crisis sanitaria ha sido la reducción de la actividad, tanto programada como urgente, en los distintos departamentos. Por ejemplo, una encuesta reciente ha constatado una reducción de en torno el 50\% en el número de coronariografías y angioplastias en España durante marzo de 2020 [27]. Como consecuencia, se espera un repunte de incidencia de otras patologías en los meses siguientes a la situación de confinamiento. Por ello es importante conocer las recomendaciones que han publicado las sociedades científicas sobre la atención al paciente con o sin sospecha de coronavirus.

En el caso de la Sociedad Española de Cardiología se han publicado, entre otros, documentos sobre la realización de estudios de imagen, gestión de procedimientos invasivos, consideraciones sobre el abordaje invasivo de la cardiopatía isquémica y estructural, y atención al paciente con insuficiencia cardiaca [28-31].

De ellos se pueden sintetizar algunos mensajes importantes, dirigidos a la protección del paciente y del profesional sanitario:

- Es esencial advertir a los pacientes sobre la conveniencia de solicitar atención médica o acudir a urgencias en casos de síntomas graves o de nueva aparición.

- $\quad$ Si bien se ha aconsejado diferir procedimientos electivos, el manejo invasivo continúa siendo de elección en el síndrome coronario agudo de alto riesgo y en el infarto con elevación del ST, siempre que las condiciones del paciente permitan el traslado a la sala de hemodinámica.

- Con el fin de reducir la exposición del paciente cardiológico, durante el brote epidémico se recomienda minimizar las consultas presenciales y las pruebas no imprescindibles en pacientes asintomáticos o con sintomatología estable. Es útil la información aportada desde el domicilio (presión arterial, peso, frecuencia cardiaca, distancia recorrida), así como la revisión en remoto de los dispositivos implantados. Es razonable realizar una analítica en pacientes que reciban modificaciones de determinados tratamientos.

- Se recomienda la máxima coordinación para minimizar tiempos de espera previos o posteriores a una consulta o procedimiento. Asimismo, se recomienda el alta precoz tras los procedimientos invasivos, siempre que esto sea posible.

- Previo al inicio de procedimientos diagnósticos o invasivos, se debe interrogar a todos los pacientes por síntomas respiratorios, mialgias, fiebre o contactos cercanos, así como tomar la temperatura corporal. 
- Los pacientes que van a recibir un procedimiento deben usar mascarilla quirúrgica. Las mascarillas FFP2 son eficaces para la protección personal, pero no sirven de barrera para la emisión de partículas, por lo que no previenen la transmisión por el paciente infectado.

- En cuanto a la protección del personal, se recomienda utilizar mascarilla quirúrgica y guantes de nitrilo en procedimientos no invasivos de bajo riesgo. En procedimientos invasivos sin sospecha de infección se aconseja bata estéril, guantes estériles, gafas antisalpicadura, gorro y mascarilla quirúrgica. En sospecha de infección o procedimientos con riesgo alto de aerosoles (por ejemplo, ecocardiografía transesofágica), se aconseja mascarilla FFP2 y bata reforzada impermeable además de los anteriores.

- Actualmente muchos centros están implementando la PCR nasofaríngea de rutina 48 horas antes de procedimientos programados, para extremar las medidas preventivas y por su posible repercusión de cara a programación o gestión de los recursos hospitalarios.

\section{Conclusiones y aspectos a recordar}

En la fisiopatología de la infección por SARS- CoV-2, la participación del sistema cardiovascular cobra un papel protagonista. La comorbilidad cardiovascular es la más común entre los pacientes infectados, y su presencia se asocia a resultados clínicos adversos. Es importante conocer las manifestaciones cardiovasculares de COVID-19, así como los potenciales efectos adversos de los tratamientos. Los años venideros revelarán los efectos sobre la salud de esta dramática pandemia, y nuestros esfuerzos deberán están centrados en la protección cardiovascular de nuestros pacientes.

Agradecimientos: A todos los profesionales sanitarios del Hospital Central de la Defensa, que han demostrado altas dosis de humildad, dedicación, compañerismo y resistencia en la lucha contra el enemigo invisible.

Contribución de los autores: Todos los autores han participado en la revisión bibliográfica, redacción y revisión crítica del manuscrito.

Conflictos de Intereses: Los autores no declaran conflicto de intereses.

\section{Abreviaturas}

Las siguientes abreviaturas son usadas en este manuscrito:

ACE2: receptor tipo 2 de la enzima convertidora de angiotensina 2

ACOD: anticoagulantes orales de acción directa

ARA II: antagonistas de los receptores de angiotensina II

COVID-19: enfermedad por coronavirus de 2019

ECG: electrocardiograma

ECV: enfermedades cardiovasculares

HBPM: heparina de bajo peso molecular

hs-cTnI: troponina I de alta sensibilidad

HTA: hipertensión arterial

IECA: inhibidores de la enzima convertidora de angiotensina

MINOCA: infarto de miocardio sin lesiones coronarias obstructivas

PCR: reacción en cadena de la polimerasa

QTc: QT corregido

SARS-CoV-2: coronavirus 2 del síndrome respiratorio agudo grave 


\section{Referencias Bibliográficas}

1. WHO Coronavirus disease (COVID-19) Situation Report - 109 . Disponible en: https://www.who.int/docs/default-source/coronaviruse/situation-reports/20200508covid-19-sitrep109.pdf?sfvrsn=68f2c632_6

2. Guan WJ, Ni ZY, Hu Y, Liang WH, Ou CQ, He JX, et al. Clinical Characteristics of Coronavirus Disease 2019 in China. N Engl J Med. 2020;382(18):1708-1720.

3. Zhou F, Yu T, Du R, Fan G, Liu Y, Liu Z, et al. Clinical course and risk factors for mortality of adult inpatients with COVID-19 in Wuhan, China: a retrospective cohort study. Lancet 2020; 395: 1054-62.

4. Guzik TJ, Mohiddin SA, Dimarco A, Patel V, Savvatis K, Marelli-Berg VM, et al. COVID-19 and the cardiovascular system: implications for risk assessment, diagnosis, and treatment options. Cardiovasc Res. 2020;cvaa106. doi:10.1093/cvr/cvaa106

5. Zhou P, Yang XL, Wang XG, Hu B, Zhang L, Zhang W, et al. A pneumonia outbreak associated with a new coronavirus of probable bat origin. Nature 2020;579:270-273.

6. Zhao S, Lin Q, Ran J, Musa SS, Yang G, Wang W, et al. Preliminary estimation of the basic reproduction number of novel coronavirus (2019-nCoV) in China, from 2019 to 2020: a data-driven analysis in the early phase of the outbreak. Int J Infect Dis 2020;92:214-217.

7. Clerkin KJ, Fried JA, Raikhelkar J, Sayer G, Griffin JM, Masoumi A, et al Coronavirus Disease 2019 (COVID-19) and Cardiovascular Disease. Circulation. 2020;10.1161/CIRCULATIONAHA.120.046941. doi:10.1161/CIRCULATIONAHA.120.046941

8. Tikellis C, Thomas MC. Angiotensin-Converting Enzyme 2 (ACE2) Is a Key Modulator of the Renin Angiotensin System in Health and Disease. Int J Pept. 2012;2012:256294.

9. Lauer SA, Grantz KH, Bi Q, Jones FK, Zheng Q, Meredith HR, et al. The Incubation Period of Coronavirus Disease 2019 (COVID-19) From Publicly Reported Confirmed Cases: Estimation and Application. Ann Intern Med. 2020;M20-0504. doi:10.7326/M20-050410.

10. Huang C, Wang Y, Li X, Ren L, Zhao J, Hu Y, et al. Clinical features of patients infected with 2019 novel coronavirus in Wuhan, China. Lancet 2020;395:497-506

11. Wang D, Hu B, Hu C, Zhu F, Liu X, Zhang J, et al. Clinical Characteristics of 138 Hospitalized Patients With 2019 Novel Coronavirus-Infected Pneumonia in Wuhan, China. JAMA. 2020;323(11):1061-1069. doi:10.1001/jama.2020.1585.

12. Mehra MR, Desai SS, Kuy S, Henry TD, Patel AN. Cardiovascular Disease, Drug Therapy, and Mortality in Covid-19. N Engl J Med. 2020;NEJMoa2007621. doi:10.1056/NEJMoa2007621.

13. Guo T, Fan Y, Chen M, Wu X, Zhang L, He T, et al. Cardiovascular Implications of Fatal Outcomes of Patients With Coronavirus Disease 2019 (COVID-19). JAMA Cardiol. 2020;e201017. doi:10.1001/jamacardio.2020.1017

14. Novel Coronavirus Pneumonia Emergency Response Epidemiology Team. The epidemiological characteristics of an outbreak of 2019 novel coronavirus diseases (COVID-19) in China. Zhonghua Liu Xing Bing Xue Za Zhi. 2020;41(2):145-151.

15. Beaney T, Burrell LM, Castillo RR, Charchar FJ, Cro S, Damasceno A, et al. May Measurement Month 2018: a pragmatic global screening campaign to raise awareness of blood pressure by the International Society of Hypertension. Eur Heart J. 2019;40(25):2006-2017.

16. Kochi AN, Tagliari AP, Forleo GB, Fassini GM, Tondo C. Cardiac and arrhythmic complications in patients with COVID-19. J Cardiovasc Electrophysiol. 2020;31(5):1003-1008.

17. Inciardi RM, Lupi L, Zaccone G, Italia L, Raffo M, Tomasoni D, et al. Cardiac Involvement in a Patient With Coronavirus Disease 2019 (COVID-19). JAMA Cardiol. 2020;10.1001/jamacardio.2020.1096. doi:10.1001/jamacardio.2020.1096

18. Hu H, Ma F, Wei X, Fang Y. Coronavirus fulminant myocarditis saved with glucocorticoid and human immunoglobulin. Eur Heart J. 2020;ehaa190. doi:10.1093/eurheartj/ehaa190

19. Magro C, Mulvey JJ, Berlin D, Nuovo G, Salvatore S, Harp J, Baxter-Stoltzfus A, Laurence J. Complement associated microvascular injury and thrombosis in the pathogenesis of severe COVID-19 infection: a report of five cases. Transl Res. 2020;S1931-5244(20)30070-0. doi:10.1016/j.trs1.2020.04.007

20. Wu Q, Zhou L, Sun X, Yan Z, Hu C, Wu J, et al. Altered Lipid Metabolism in Recovered SARS Patients Twelve Years after Infection. Sci Rep. 2017;7(1):9110. 
21. Driggin E, Madhavan MV, Bikdeli B, Chuich T, Laracy J, Biondi-Zoccai G, et al. Cardiovascular Considerations for Patients, Health Care Workers, and Health Systems During the COVID-19 Pandemic. J Am Coll Cardiol. 2020;75(18):2352-2371.

22. Wu CI, Postema PG, Arbelo E, Behr ER, Bezzina CR, Napolitano C, et al. SARS-CoV-2, COVID-19, and inherited arrhythmia syndromes. Heart Rhythm. 2020:S1547-5271(20)30285-X. doi: 10.1016/j.hrthm.2020.03.024.

23. Tisdale JE, Jaynes HA, Kingery JR, Mourad NA, Trujillo TN, Overholser BR, et al. Development and validation of a risk score to predict QT interval prolongation in hospitalized patients. Circ Cardiovasc Qual Outcomes. 2013;6(4):479-87.

24. Reynolds HR, Adhikari S, Pulgarin C, Troxel AB, Iturrate E, Johnson SB, et al. Renin-AngiotensinAldosterone System Inhibitors and Risk of Covid-19. N Engl J Med. 2020:NEJMoa2008975. doi: 10.1056/NEJMoa2008975.

25. Zhang P, Zhu L, Cai J, Lei F, Qin JJ, Xie J, et al. Association of Inpatient Use of Angiotensin Converting Enzyme Inhibitors and Angiotensin II Receptor Blockers with Mortality Among Patients With Hypertension Hospitalized With COVID-19. Circ Res. 2020. doi: 10.1161/CIRCRESAHA.120.317134.

26. Vivas D, Roldán V, Esteve-Pastor MA, Roldán I, Tello-Montoliu A, Ruiz-Nodar JM, et al. Recomendaciones sobre el tratamiento antitrombótico durante la pandemia COVID-19. Posicionamiento del Grupo de Trabajo de Trombosis Cardiovascular de la Sociedad Española de Cardiología. Rev Esp Cardiol. 2020. doi: 10.1016/j.recesp.2020.04.006.

27. Rodríguez-Leor O, Cid-Álvarez B, Ojeda S, Martín-Moreiras J, Ramón Rumoroso J, López-Palop R, et al. Impacto de la pandemia de COVID-19 sobre la actividad asistencial en cardiología intervencionista en España. REC interventional cardiology. 2020. DOI:10.24875/RECIC.M20000120

28. Juan Bagudá J, Farrero Torres M, García-Cosío MD, Recio Mayoral A, Sabé Fernández N, García Pinilla JM, et al. Implicaciones de la pandemia por COVID-19 para el paciente con insuficiencia cardiaca, trasplante cardiaco y asistencia ventricular. Recomendaciones de la Asociación de Insuficiencia Cardiaca de la Sociedad Española de Cardiología. REC: CardioClinics. 2020. doi:10.1016/j.rccl.2020.03.007

29. Recomendaciones para la realización de estudios de imagen cardiaca durante el brote de coronavirus COVID-19. Disponible en: https://secardiologia.es/imagen/formacion/documentos/11450recomendaciones-para-la-realizacion-de-estudios-de-imagen-cardiaca-durante-el-brote-de-coronaviruscovid-19

30. Romaguera R, Cruz-González I, Ojeda S, Jiménez-Candil J, Calvo D, García Seara J, et al. Gestión de las salas de procedimientos invasivos cardiológicos durante el brote de coronavirus COVID-19. Documento de consenso de la Asociación de Cardiología Intervencionista y la Asociación del Ritmo Cardiaco de la Sociedad Española de Cardiología. REC interventional cardiology. 2020. DOI:10.24875/RECIC.M20000114

31. Romaguera R, Cruz-González I, Jurado-Román A, Ojeda S, Fernández-Cisnal A, Jorge-Pérez P, et al. Consideraciones sobre el abordaje invasivo de la cardiopatía isquémica y estructural durante el brote de coronavirus COVID-19. REC interventional cardiology. 2020. DOI:10.24875/RECIC.M20000119

(C) 2020 por los autores; Esta obra está sujeta a la licencia de Reconocimiento 4.0 Internacional de Creative Commons. Para ver una copia de esta licencia, visite http://creativecommons.org/licenses/by-nc-nd/4.0/. 